\title{
ESTUDO DO EQUILÍBRIO DA ADSORÇÃO DE NITROGÊNIO AMONIACAL DE LIXIVIADO DE ATERRO SANITÁRIO EM ZEÓLITA
}

\author{
P. A. STEINER-FERREIRA ${ }^{1}$, A. P. SCHEER ${ }^{2}$ e M.C.B. BRAGA ${ }^{1}$ \\ ${ }^{1}$ Universidade Federal do Paraná, Departamento de Hidráulica e Saneamento \\ ${ }^{2}$ Universidade Federal do Paraná, Departamento de Engenharia Química \\ e-mail para contato: patyasteiner@yahoo.com.br
}

\begin{abstract}
RESUMO - Lixiviados são caracterizados como uma solução aquosa complexa, contendo várias substâncias potencialmente tóxicas ou inibidoras de sistemas biológicos de tratamento de efluentes e, também, de processos bioquímicos que ocorrem nos corpos hídricos. Entre essas substâncias está o nitrogênio amoniacal. Para a adequação das características do lixiviado para tratamento ou descarte são necessárias etapas de pré ou de pós-tratamento como, por exemplo, a adsorção. $\mathrm{O}$ objetivo desta pesquisa foi estudar o equilíbrio do processo de adsorção de nitrogênio amoniacal de lixiviado de aterro sanitário utilizando zeólita como adsorvente. Foram realizados ensaios em batelada para a determinação da cinética de adsorção e das isotermas de Langmuir e Freundlich. O tempo de equilíbrio da adsorção do nitrogênio amoniacal do lixiviado bruto e do efluente de lagoa facultativa foi de 12 horas, para ambas isotermas. Entretanto, a isoterma de Freundlich apresentou o melhor ajuste aos resultados.
\end{abstract}

\section{INTRODUÇÃO}

As características construtivas dos aterros sanitários permitem minimizar os efeitos das duas principais fontes de poluição oriundas dos resíduos sólidos, o biogás e o lixiviado. O lixiviado é composto pela decomposição da parte orgânica dos resíduos, além de fontes líquidas externas como a drenagem superficial e a precipitação pluvial, que infiltra nas camadas das células e percola até a base do aterro para ser coletado pelo sistema de drenagem. Vários interferentes atuam na geração do lixiviado e valores elevados de $\mathrm{pH}$, de alcalinidade, de metais e de matéria matéria orgânica e inorgânica são típicos (JEONG-HONN et al., 2001; BERTAZZOLI e PELEGRINI, 2002; HASARet al., 2009; MIAO et al., 2014).

Além disso, uma característica comumente observada nos lixiviados de aterros sanitários é a elevada concentração de nitrogênio, encontrado principalmente na forma amoniacal. Altas concentrações de nitrogênio amoniacal podem ser tóxicas e inibidoras aos processos biológicos de tratamento do lixiviado, além de poder causar a eutrofização de corpos aquáticos, caso seja realizado o seu descarte sem prévio tratamento(LI e ZHAO, 2001; JOKELA et al., 2002; YUSOF et al., 2010; WIDIASTUTI et al., 2011). 


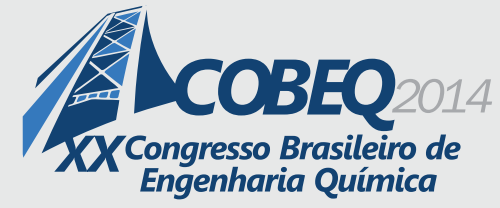

A forma mais comumente utilizada para a redução da concentração de poluentes presentes nos lixiviados são os tratamentos biológicos, porém, muitas vezes, não são suficientes para remover o nitrogênio amoniacal, sendo recomendada a sua associação a processos físico-químicos (KILIÇ et al., 2007; COTMAN e GOTVAJN, 2010). Entre os diversos processos físico-químicos utilizados para a remoção de nitrogênio amoniacal podem ser o stripping de amônia, a precipitação química e a adsorção. A adsorção apresenta vantagens por ser uma técnica simples e efetiva para tratamento de efluentes e o sucesso do uso dessa técnica depende do emprego de um adsorvente com ação efetiva (WANG e PENG, 2010).

Portanto, visando à adequação das características do lixiviado do Aterro Sanitário de Curitiba tanto para tratamento biológico como para posterior lançamento em corpo hídrico receptor, esta pesquisa estudou o equilíbrio da adsorção do nitrogênio amoniacal dos lixiviados produzidos neste aterro utilizando zeólita como adsorvente.

\section{MATERIAIS E MÉTODO}

Para a obtenção dos dados de cinética e modelagem das isotermas de adsorção foram realizados ensaios em batelada, conforme descrito a seguir.

\subsection{Determinação da cinética de adsorção}

Para a determinação da cinética de adsorção foram realizados experimentos com o lixiviado bruto (P1) e após tratamento (P2). O procedimento experimental consistiu na transferência de um volume de $100 \mathrm{~mL}$ do lixiviado para frascos Erlenmeyer contendo, aproximadamente, 3,33 g de adsorvente (zeólita). Os frascos foram vedados com três camadas de Parafilm ${ }^{\circledR}$ e, em seguida, colocados em incubadora com agitação orbital, marca Tecnal, modelo TE-421, a $25^{\circ} \mathrm{C}$ sob agitação de $50 \mathrm{rpm}$.

Nos tempos de 15 min, 30 min, 1 h, 4 h, 8 h, 12 h, 24 h e 48 h, os Erlenmeyers foram retirados do equipamento e alíquotas de $50 \mathrm{~mL}$ foram filtradas em papel filtro qualitativo (espessura de 0,205 $\mathrm{mm}$, gramatura de $80 \mathrm{~g} \mathrm{~m}-2$ e diâmetro de $12,5 \mathrm{~cm}$ ).

A massa de soluto adsorvido por massa de adsorvente em cada tempo $\left(q_{t}\right)$ foi determinada por balanço de massa, por meio da Equação (1). As alíquotas retiradas nos tempos indicados foram analisadas para a determinação da concentração de nitrogênio amoniacal de acordo com metodologia especificada pelo Standard Methods for the Examination of Water and Wastewater (APHA, 1998). A leitura foi realizada por espectrofotometria de luz visível, em espectrofotômetro SHIMADZU, modelo UV-1601PC, no comprimento de onda de 640nm, tendo sido foi utilizadas cubetas de quartzo de $5 \mathrm{~mL}$.

$q_{t}=\frac{\left(C_{0}-C_{t}\right)}{m} V$ 
em que:

$C_{0}=$ concentração inicial de adsorvato $(\mathrm{mg} / \mathrm{L})$

$C_{t}=$ concentração de adsorvato no tempo $t(\mathrm{mg} / \mathrm{L})$

$V=$ volume da batelada $(\mathrm{L})$

$m=$ massa do material adsorvente $(\mathrm{g})$

A eficiência da remoção do nitrogênio amoniacal pelo processo de adsorção foi calculada utilizando-se a Equação (2).

$\operatorname{Eficiência}(\%)=\frac{C_{\text {lix.bruto }}-C_{\text {lix.adsorv }}}{C_{\text {lix.bruto }}} \times 100$

em que:

$C_{\text {lix.bruto }}=$ Concentração do lixiviado bruto $(\mathrm{mg} / \mathrm{L})$;

$C_{\text {lix.adsor. }}=$ Concentração do lixiviado após adsorção $(\mathrm{mg} / \mathrm{L})$.

\subsection{Determinação da isoterma de adsorção}

Os experimentos para a obtenção das isotermas de adsorção do nitrogênio amoniacal foram realizados em triplicata, a $15{ }^{\circ} \mathrm{C}, 23{ }^{\circ} \mathrm{C}$ e $30^{\circ} \mathrm{C}$. Alíquotas de $100 \mathrm{~mL}$ de lixiviado P1 diluídas (1700 $\mathrm{mg} / \mathrm{L}, 1200 \mathrm{mg} / \mathrm{L}, 760 \mathrm{mg} / \mathrm{L}, 340 \mathrm{mg} / \mathrm{L}$ e $130 \mathrm{mg} / \mathrm{L})$ e $P 2$ diluídas $(700 \mathrm{mg} / \mathrm{L}, 550 \mathrm{mg} / \mathrm{L}, 450 \mathrm{mg} / \mathrm{L}$, $350 \mathrm{mg} / \mathrm{L}$ e $200 \mathrm{mg} / \mathrm{L}$ ) foram adicionados a frascos Erlenmeyer contendo 3,33 $\mathrm{g}$ de zeólita e, então, submetidas à agitação constante a $50 \mathrm{rpm}$, no tempo de equilíbrio determinado pela cinética de adsorção. A quantidade adsorvida no equilíbrio $\left(q_{e q}\right), \mathrm{em} \mathrm{mg} / \mathrm{g}$, foi determinada por balanço de massa conforme Equação (3).

$q_{e q}=\frac{\left(C_{0}-C_{e q}\right)}{m} V$

em que:

$C_{e q}=$ concentração final do adsorvato em solução no equilíbrio $(\mathrm{mg} / \mathrm{L})$

Os dados experimentais das isotermas foram modelados por meio de regressão não linear empregando-se os modelos de Langmuir e Freundlich (Equações (4) e (5), respectivamente, apresentadas a seguir.

$q_{e}=\frac{q_{m} \cdot K_{L} \cdot C_{e}}{1+K_{L} \cdot C_{e}}$

em que:

$C_{e}(\mathrm{mg} / \mathrm{L})=$ concentração do soluto presente na solução em equilíbrio

$q_{e q}(\mathrm{mg} / \mathrm{g})=$ quantidade adsorvida no equilíbrio, por unidade de massa de adsorvente 
$q_{e q}=K_{F} C_{e q}^{1 / n}$

em que:

$K_{F}\left((m g / g)(L / m g)\right.$ e $n$ são constantes dependentes da temperatura, sendo que a constante $K_{F}$ está relacionada à capacidade de adsorção do sólido e $1 / n$ indica a intensidade de adsorção. Geralmente $n$ encontra-se na faixa de 1 a 5 . A adsorção é favorável quando $1<n<10$. Assim, se o valor de $1 / n$ for menor que 1, o processo de adsorção é favorável (BULUT e AYDIN, 2006).

De posse dos dados de equilíbrio foi aplicado o parâmetro estatístico SS (Sum of Squares), calculado pela Equação (6). REVER REDAÇÃO DESTE TRECHO - QUANDO DEVE SER UTILIZADO O SS Este parâmetro fornece um forte argumento da adequação de cada modelo à descrição dos dados. Quanto menor o valor de $S S$, melhor será o ajuste.

$$
S S=\frac{1}{n} \sqrt{\sum_{i=1}^{n}\left(q_{\text {teórico }}-q_{\text {exp erimentass }}\right)^{2}}
$$

em que:

$S S=$ Soma dos quadrados

$n=$ número de medidas utilizadas no cálculo

$q_{\text {teórico }}=q$ do ajuste

$q_{\text {experimental }}=q$ obtido experimentalmente

\section{APRESENTAÇÃO E DISCUSSÃO DOS RESULTADOS}

\subsection{Cinética do processo de adsorção}

Para determinar o tempo de equilíbrio da adsorção foram realizados os experimentos de cinética de adsorção com os lixiviados de P1 e de P2, cujos resultados são apresentados na Tabela 1 e Figura 1.

A partir dos dados apresentados na Tabela 1 e na Figura 1 foi possível determinar que o tempo de equilíbrio da adsorção do nitrogênio amoniacal em zeólita com o lixiviado foi de 12 horas (720 minutos) para P1 e para P2.

A diferença entre os valores de capacidade de adsorção e de eficiência é devida à diferença nas composições químicas de cada lixiviado. Enquanto P1 é o lixiviado bruto, ou seja, que acabou de ser gerado, contendo mais compostos e em maior concentração, P2 é o efluente que já possui um tratamento prévio (lagoas aeradas e lagoa facultativa), ou seja, uma carga menor de poluentes. 
Tabela 1 - Capacidade de adsorção e eficiência de remoção de nitrogênio amoniacal em zeólita

\begin{tabular}{|c|c|c|c|c|}
\cline { 2 - 5 } \multicolumn{1}{c|}{} & \multicolumn{2}{c|}{ Lixiviado Bruto (P1) } & \multicolumn{2}{c|}{ Lixiviado Tratado (P2) $^{\mathbf{c}}$} \\
\hline Tempo (min) & $\boldsymbol{q}^{\mathbf{a}}$ & Efic. $(\boldsymbol{\%})$ & $\boldsymbol{q}^{\mathbf{a}}$ & Efic. $(\boldsymbol{\%})$ \\
\hline $\mathbf{1 5}$ & $2,17 \pm 0,3$ & $4,4 \pm 0,5$ & $1,45 \pm 0,4$ & $7,6 \pm 1,3$ \\
\hline $\mathbf{3 0}$ & $4,98 \pm 1,1$ & $10,2 \pm 2,1$ & $1,65 \pm 0,5$ & $8,6 \pm 1,6$ \\
\hline $\mathbf{6 0}$ & $6,13 \pm 0,4$ & $12,5 \pm 0,8$ & $1,97 \pm 0,3$ & $10,4 \pm 0,7$ \\
\hline $\mathbf{2 4 0}$ & $6,70 \pm 1,5$ & $13,7 \pm 2,5$ & $2,94 \pm 0,4$ & $15,4 \pm 1,5$ \\
\hline $\mathbf{4 8 0}$ & $7,42 \pm 0,8$ & $15,1 \pm 1,7$ & $3,76 \pm 0,5$ & $19,7 \pm 2,1$ \\
\hline $\mathbf{7 2 0}$ & $9,65 \pm 0,9$ & $19,7 \pm 1,2$ & $5,14 \pm 0,6$ & $27,0 \pm 1,8$ \\
\hline $\mathbf{1 4 4 0}$ & $10,65 \pm 1,0$ & $21,8 \pm 2,1$ & $5,78 \pm 0,6$ & $30,3 \pm 2,9$ \\
\hline $\mathbf{2 8 8 0}$ & $13,00 \pm 1,0$ & $26,6 \pm 5,4$ & $6,88 \pm 0,7$ & $33,7 \pm 3,5$ \\
\hline
\end{tabular}

${ }^{\mathrm{a}} q$ médio: capacidade de adsorção $\left(\mathrm{mg}_{\text {adsorvato }} / \mathrm{g}_{\text {adsorvente }}\right)$

${ }^{b}$ Conc. Inicial: $1632,25 \mathrm{mg} \mathrm{N}-\mathrm{NH}_{3} / \mathrm{L}$

${ }^{c}$ Conc. Inicial: 634,91 mg N-NH $3 / \mathrm{L}$

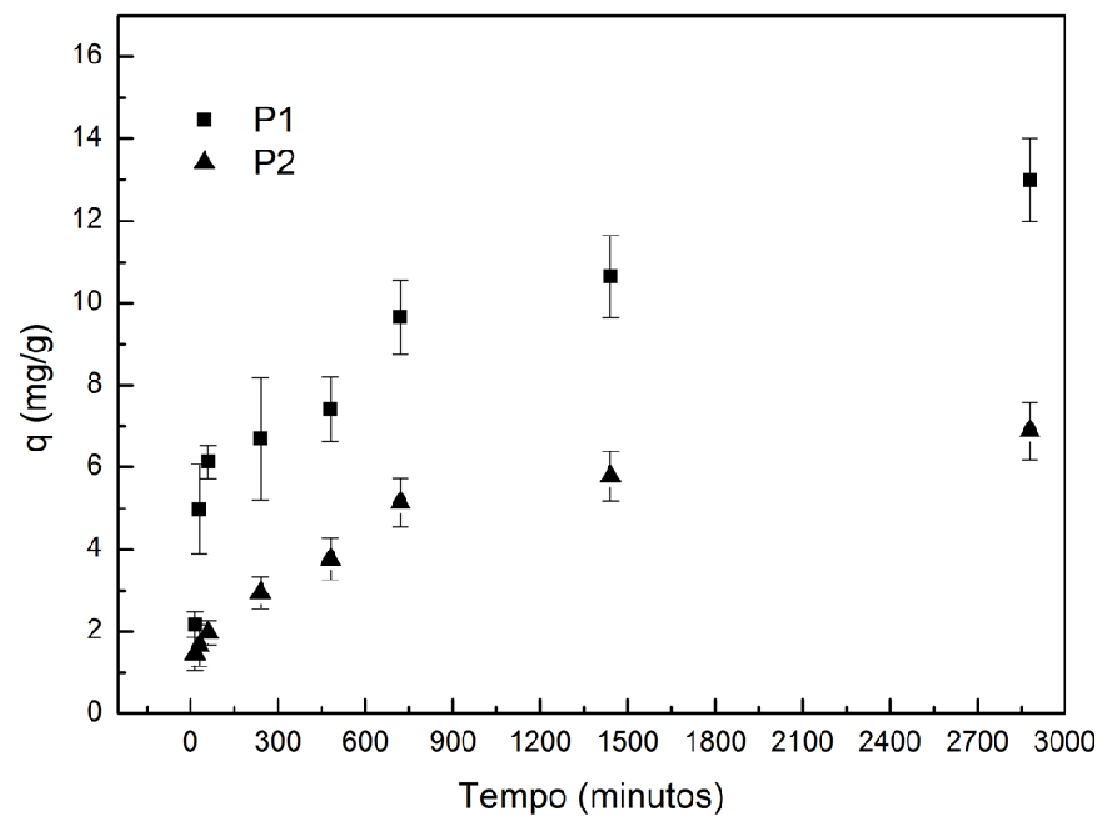

Figura 1 - Capacidade de adsorção de nitrogênio amoniacal em zeólita

\subsection{Isotermas do processo de adsorção}

O tempo de realização dos experimentos foi igual ao tempo de equilíbrio determinado pelos experimentos de cinética. Este tempo foi de 12 horas, entretanto, para garantir que o equilíbrio tivesse sido alcançado, o tempo de duração do experimento foi extendido até 16 horas. Nas Tabelas 2 e 3 são apresentados os resultados obtidos. 
Tabela 2 - Capacidade adsortiva de P1 a $15^{\circ} \mathrm{C}, 23{ }^{\circ} \mathrm{C}$ e $30^{\circ} \mathrm{C}$

\begin{tabular}{|c|c|c|c|}
\cline { 2 - 4 } \multicolumn{1}{c|}{} & \multicolumn{3}{c|}{ Temperatura $\left({ }^{\mathbf{o}} \mathbf{C}\right)$} \\
\hline Concentração $\left(\mathbf{m g ~ N}-\mathbf{N H}_{\mathbf{3}} / \mathbf{L}\right)$ & $\mathbf{1 5}$ & $\mathbf{2 3}$ & $\mathbf{3 0}$ \\
\hline $\mathbf{1 3 0}$ & $1,82 \pm 0,35$ & $2,74 \pm 0,09$ & $2,63 \pm 0,06$ \\
\hline $\mathbf{3 4 0}$ & $6,88 \pm 0,71$ & $8,21 \pm 0,45$ & $8,39 \pm 0,60$ \\
\hline $\mathbf{7 6 0}$ & $9,55 \pm 1,31$ & $13,55 \pm 2,22$ & $11,52 \pm 3,01$ \\
\hline $\mathbf{1 2 0 0}$ & $11,00 \pm 2,12$ & $14,00 \pm 2,15$ & $13,00 \pm 2,58$ \\
\hline $\mathbf{1 7 0 0}$ & $16,18 \pm 0,60$ & $15,94 \pm 1,26$ & $15,41 \pm 1,8$ \\
\hline
\end{tabular}

Tabela 3 - Capacidade adsortiva de $\mathrm{P} 2$ a $15^{\circ} \mathrm{C}, 23{ }^{\circ} \mathrm{C}$ e $30^{\circ} \mathrm{C}$

\begin{tabular}{|c|c|c|c|}
\cline { 2 - 4 } \multicolumn{1}{c|}{} & \multicolumn{3}{c|}{ Temperatura $\left({ }^{\mathbf{o}} \mathbf{C}\right)$} \\
\hline Concentração $\left(\mathbf{m g ~ N}-\mathbf{N H}_{\mathbf{3}} / \mathbf{L}\right)$ & $\mathbf{1 5}$ & $\mathbf{2 3}$ & $\mathbf{3 0}$ \\
\hline 200 & $0,22 \pm 1,12$ & $0,80 \pm 0,14$ & $0,77 \pm 0,15$ \\
\hline 350 & $3,08 \pm 0,24$ & $3,68 \pm 0,19$ & $3,12 \pm 0,25$ \\
\hline 450 & $3,69 \pm 0,52$ & $4,92 \pm 0,19$ & $5,22 \pm 0,95$ \\
\hline 550 & $4,76 \pm 0,33$ & $5,23 \pm 0,51$ & $6,02 \pm 0,38$ \\
\hline 700 & $6,42 \pm 0,21$ & $7,27 \pm 0,65$ & $7,89 \pm 0,17$ \\
\hline
\end{tabular}

A análise dos dados experimentais utilizados para a obtenção das isotermas indica que a capacidade adsorvida aumenta com o aumento da temperatura e com a concentração inicial.Neste estudo foram avaliadas as isotermas de Langmuir e Freundlich nas três temperaturas analisadas $\left(15^{\circ} \mathrm{C}, 23^{\circ} \mathrm{C}\right.$ e $\left.30^{\circ} \mathrm{C}\right)$. Na Tabela 4 , são apresentados os resultados para os parâmetros das isotermas ajustadas aos dados experimentais.

Tabela 4 - Parâmetros dos modelos de isotermas

\begin{tabular}{|c|c|c|c|c|c|c|c|c|}
\cline { 3 - 8 } \multicolumn{2}{c}{} & \multicolumn{6}{c|}{ P1 } & \multicolumn{4}{c|}{ P2 } \\
\cline { 3 - 8 } \multicolumn{2}{c|}{} & \multicolumn{6}{c|}{ Tempertura $\left({ }^{\mathbf{0}} \mathbf{C}\right)$} \\
\hline Isoterma & Parâmetro & $\mathbf{1 5}$ & $\mathbf{2 3}$ & $\mathbf{3 0}$ & $\mathbf{1 5}$ & $\mathbf{2 3}$ & $\mathbf{3 0}$ \\
\hline Langmuir & $\mathrm{R}^{\mathbf{2}}$ & 0,98 & 0,97 & 0,99 & 0,93 & 0,93 & 0,96 \\
\hline Freundlich & $\mathrm{R}^{\mathbf{2}}$ & 0,97 & 0,98 & 0,99 & 0,93 & 0,93 & 0,96 \\
\hline
\end{tabular}

Nota: $\mathrm{R}^{2}=$ coeficiente de correlação

Como pode ser observado na Tabela 4, valores para o coeficiente de correlação $\left(\mathrm{R}^{2}\right)$ para as duas isotermas são semelhantes, para as três temperaturas. Assim, foi necessário aplicar o método estatístico SS para determinar a isoterma que melhor se adequava aos dados experimentais. Na Tabela 5 são apresentados os resultados da aplicação da ferramenta estatística. 
Tabela 5 - Valores de SS para cada um dos modelos e temperaturas estudados

\begin{tabular}{|c|c|c|c|c|c|c|}
\cline { 2 - 7 } \multicolumn{1}{c|}{} & \multicolumn{9}{c|}{ P1 } & \multicolumn{4}{c|}{ P2 } \\
\cline { 2 - 7 } \multicolumn{1}{c|}{} & \multicolumn{6}{c|}{ TEMPERATURA $\left({ }^{\circ} \mathbf{C}\right)$} \\
\hline MODELO & $\mathbf{1 5}$ & $\mathbf{2 3}$ & $\mathbf{3 0}$ & $\mathbf{1 5}$ & $\mathbf{2 3}$ & $\mathbf{3 0}$ \\
\hline Langmuir & 0,46 & 0,34 & 0,19 & 0,24 & 0,25 & 0,22 \\
\hline Freundlich & 0,35 & 0,25 & 0,10 & 0,20 & 0,21 & 0,20 \\
\hline
\end{tabular}

Assim, como pode ser observado, para a zeólita, o modelo da isoterma de Freundlich ajustou-se melhor aos dados experimentais para os lixiviados de P1 e P2. Isso significa dizer que não é possível prever o estado de saturação do processo de adsorção. Desta forma, a adsorção pode ser considerada em várias camadas (REED e MATSUMOTO, 1993).

\section{CONSIDERAÇÕES FINAIS}

Dos resultados obtidos, é possível afirmar que o tempo de equilíbrio de adsorção do nitrogênio amoniacal de lixiviado do Aterro Sanitário de Curitiba em zeólita é de 12 horas, ou seja, a partir deste tempo não haverá aumento da capacidade de adsorção nem da eficiência de remoção de nitrogênio amoniacal. A eficiência máxima de remoção de nitrogênio amoniacal foi de, aproximadamente, $27 \%$ para P1 e $34 \%$ para P2. Em função dos valores obtidos no método estatístico SS pode-se deduzir que o modelo da isoterma de Freundlich foi aquele mais se adequou aos dados experimentais, indicando que o processo de adsorção pode ocorrer em várias camadas.

\section{REFERENCIAS}

APHA; AWWA e WEF.Standard Methods for the Examination of Water and Wastewater.20th Edition.Washington: APHA, AWWA, WEF, 1998.

BERTAZZOLI, R.; PELEGRINI, R. Photoelectrochemical discoloration and degrationpf organic pollutants in aqueous solution. Quím. Nova, v. 25, n. 3, p. 447-482, 2002.

BULUT, Y; AYDIN, H. A kinetics and thernodynamics study of methylene blue adsorption on wheat shells. Desalin., v. 194, p. 259 - 267, 2006.

COTMAN, M., GOTVAJN, A. Z., Comparison of different physic-chemical methods for the removal of toxicants from landfill leachate. J Hazard. Mat. v. 178, p. 298-305, 2010. 


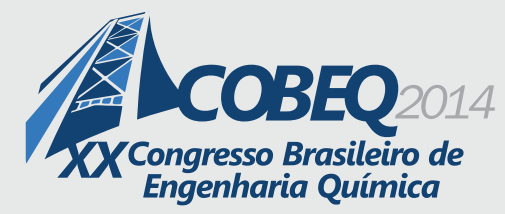

19 a 22 de outubro de 2014

Florianópolis/SC

HASAR, H.; UNSAL, S.A.; IPEK, U.; KARATAS, S.; CINAR, O.; YAMAN, C.; KINACI, C. Stripping/flocculation/membrane bioreactor/reverse osmosis treatment of municipal landfill leachate. J. Hazard. Mat, .v.171 p. 309-310, 2009.

JEONG-HONN, I. M.; HAE-JIN, W.; MYUNG-WON, C.; KI-BACK, H.; CHANG-WON, K. Simultaneous organic and nitrogen removal from municipal landfill leachate using an anaerobicaerobic system. Water Res., v. 35, n. 10, p. 2043 - 2410, 2001.

JOKELA, J. P. Y.; KETTUNEN, R. H.; SORMUNEN, K. M.; RINTALA, J. A. Biological nitrogen removal from municipal landfill leachate: low-cost nitrification in biofilters and laboratory scale in-situ denitrification. Water Res. v. 36, n. 16, p. 4079-4087, 2002.

KILIÇ, M.Y., KESTIOGLU K., YONAR, T. Landfill leachate treatment by the combination of physicochemical methods with adsorption process. J. Biol. Environ. Sci., v.1, n.1, p.37-43, 2007.

LI, X.Z.; ZHAO, Q.L. Efficiency of biological treatment affected by high strength of ammonium-nitrogen as pretreatment, Chemosphere, p.v. 44, p. 37-43, 2001.

MIAO, L.; WANG, K.; WANG, S.; ZHU, R.; LI, B.; PENG, Y.; WENG, D. Advanced nitrogen removal from landfill leachate using real-time controlled three-stage sequence batch reactor (SBR) system. Biores. Technol., v.159, p. 258-265, 2014.

REED B. E.; MATSUMOTO M. R. Modeling cadmium adsorption by actived carbon using the Langmuir and Freundlich isotherm expressions. Sep. Sc. Technol., v. 28, n. 13 e 14, p. 2179 2195, 1993.

YUSOF, N.; HASSAN, M.A.; PHANG,L.Y.; TABATABAEI, M.; OTHMAN,M.R.; MORI, M.; WAKISAKA, M.; SAKAI, K.; SHIRAI, Y. Nitrification of ammonium-rich sanitary landfill leachate. Waste Manag. v. 30, p. 100- 109, 2010.

WANG, S.; PENG, Y. Natural zeolites as effective adsorbents in water and wastewater treatment. Chem. Eng. J., v.156, p. 11-24, 2010.

WIDIASTUTI, N.; WU, H.; ANG, H. M.; ZHANG, D. Removal of ammonium from greywater using natural zeolite. Desal., v.277, p. 15-23, 2011. 